\title{
A Multivariate Grey Prediction Model Using Neural Networks with Application to Carbon Dioxide Emissions Forecasting
}

\author{
Yu-Jing Chiu $\mathbb{D}^{1},{ }^{1}$ Yi-Chung Hu $\mathbb{D}^{1},{ }^{1}$ Peng Jiang $\mathbb{D}^{2},{ }^{2}$ Jingci Xie $\mathbb{D}^{3},{ }^{3}$ and Yen-Wei Ken $\mathbb{D}^{1}$ \\ ${ }^{1}$ Department of Business Administration, Chung Yuan Christian University, Taoyuan City, Taiwan \\ ${ }^{2}$ School of Business, Shandong University, Weihai, China \\ ${ }^{3}$ School of Management, Shandong University, Jinan, China \\ Correspondence should be addressed to Jingci Xie; xjc@sdu.edu.cn
}

Received 8 September 2020; Revised 10 October 2020; Accepted 12 October 2020; Published 29 October 2020

Academic Editor: Tomas Balezentis

Copyright (c) $2020 \mathrm{Yu}$-Jing Chiu et al. This is an open access article distributed under the Creative Commons Attribution License, which permits unrestricted use, distribution, and reproduction in any medium, provided the original work is properly cited.

The forecast of carbon dioxide $\left(\mathrm{CO}_{2}\right)$ emissions has played a significant role in drawing up energy development policies for individual countries. Since data about $\mathrm{CO}_{2}$ emissions are often limited and do not conform to the usual statistical assumptions, this study attempts to develop a novel multivariate grey prediction model (MGPM) for $\mathrm{CO}_{2}$ emissions. Compared with other MGPMs, the proposed model has several distinctive features. First, both feature selection and residual modification are considered to improve prediction accuracy. For the former, grey relational analysis is used to filter out the irrelevant features that have weaker relevance with $\mathrm{CO}_{2}$ emissions. For the latter, predicted values obtained from the proposed MGPM are further adjusted by establishing a neural-network-based residual model. Prediction accuracies of the proposed MGPM were verified using real $\mathrm{CO}_{2}$ emission cases. Experimental results demonstrated that the proposed MGPM performed well compared with other MGPMs considered.

\section{Introduction}

Carbon dioxide $\left(\mathrm{CO}_{2}\right)$ is mainly produced from fossil fuel combustion [1], and reducing the impact that energy consumption and economic growth have on $\mathrm{CO}_{2}$ emissions has become a global challenge [2]. According to the International Energy Agency (IEA) [3], total emissions of greenhouse gas in 2018 were a record 33.1 billion tons, along with a global economic growth rate that increased by 3.2\%. Despite a $\mathrm{CO}_{2}$ emission plateau from 2014 to 2016, the IEA reported that China and USA were the highest energy-using and carbon-emitting countries, and $\mathrm{CO}_{2}$ emissions went up in each country by $2.5 \%$ and $3.1 \%$, respectively, mainly arising from an increased use of fossil fuel to meet the energy demand. In fact, $\mathrm{CO}_{2}$ emissions can significantly give rise to climate change and have a negative impact on economic growth. Therefore, to keep a green economic growth, the national authorities make an effort to devise energy development policies that reduce the impact of $\mathrm{CO}_{2}$ emissions.
An accurate forecast of $\mathrm{CO}_{2}$ emissions becomes a remarkable issue when public sectors set up policies.

From the viewpoint of the grey system theory [4-6], the prediction of $\mathrm{CO}_{2}$ emissions can be viewed as a grey system problem because although the relevant features, such as energy consumption, population, and gross domestic product (GDP) [7-9], influence $\mathrm{CO}_{2}$ emissions, the precise relationship between these features and emissions is not clear. Furthermore, it is possible that emissions data do not conform to any statistical assumptions [7]. Compared with the prediction models implemented by artificial intelligence techniques [10-15], statistical models including logistic models [16], multivariate regression [17, 18], and time series analysis $[19,20]$, MGPMs have the advantage of characterizing an unknown system using limited samples [6], without requiring conformance with statistical assumptions. Despite huge amount of data we can collect, only a few sample data points are required to achieve reliable and acceptable prediction accuracy $[21,22]$. Therefore, it is 
interesting to apply the multivariate grey prediction models (MGPMs) to $\mathrm{CO}_{2}$ emissions.

In contrast to the frequently used GM $(1,1)$, the firstorder grey differential equation with one variable, which does not consider the influence of relevant factors on the system [23], the GM $(1, N)$, which consists of a system's characteristic sequence and several relevant factor sequences, is fundamental to MGPMs and has been widely applied to time series forecasting, such as the forecasting of traffic flow [24], number of motor vehicles [25], production in high-tech industries [26, 27], energy consumption $[28,29]$, integrated circuit output $[30,31]$, OFDI analysis [32], and pattern classification [33, 34]. Many GM $(1, N)$ variants have been introduced to improve the prediction accuracy of the traditional GM $(1, N)$, such as a rolling multivariable model [8], background value optimization [27], the optimization of GM $(1, N)(\operatorname{OGM}(1, N))$ using grey differential equations with linear correction and grey quantity terms $[25,35]$, nonlinear grey models $\operatorname{NGM}(1, N))$ using grey differential equations with power exponents, and transformed NGM $(1, N)$ (TNGM $(1, N)$ ) [28]. These MGPMs have shown their superior prediction performance when used with time series problems.

Grey prediction has demonstrated its effectiveness on $\mathrm{CO}_{2}$ emissions forecasting, such as univariate grey prediction models by Pao et al. [36], Wu et al. [2], and Xu et al. [37], prediction models based on trends of driving coefficients (TDVGM $(1, N)$ ) by Ding et al. [7], TNGM $(1, N)$ by Wang and Ye [28], multikernel nonlinear multivariable grey model by Duan et al. [23], a forecasting method for the trafficrelated emissions by Xie et al. [38], a nonlinear grey power model (DGPM $(1, N))$ by Ding et al. [39], and a nonequigap grey Verhulst model by Wang and Li [40]. This study contributed to developing a distinctive MGPM with feature selection and residual modification performed to improve prediction accuracy. For the former, since system performance can be improved by feature selection [41], the proposed MGPM performs grey relational analysis (GRA) $[4-6,42]$ to estimate relevance between independent variables and $\mathrm{CO}_{2}$ emissions. For the latter, it has been suggested that the prediction accuracy of the GM $(1,1)$ can be improved by residual modification [4]. However, it is very interesting to extend residual modification to the GM $(1, N)$. A neural-network-based residual model is thus created for the proposed MGPM to adjust predicted values from the GM $(1, N)$. To sum up, the proposed MGPM with feature selection and residual modification can be treated as a residual modification model. Genetic algorithms (GAs) are employed to determine required parameters of the GRA and GM $(1, N)$ to construct the proposed MGPM with high prediction accuracy. Experimental results have indicated that the proposed MGPM performs well compared with the other MGPMs considered. It is noted that, to estimate the correlation between the system behavior variable and the influential factors, the proposed prediction model used GA to determine a threshold value that is not easily prespecified. In contrast, Xie et al. [38] applied GRA to identify relevant factors from passenger cars per 1000 inhabitants, stock of vehicles, volume of freight transport relative to GDP
(VFTRG), and volume of passenger transport relative to GDP, but only VFTRG (with time lags four) with maximum correlation was considered. Meanwhile Ding et al. [39] used a prespecified threshold value to evaluate the relationship among factors.

The remainder of the paper is organized as follows. Section 2 introduces the traditional GM $(1, N)$, grey residual modification model, and Section 3 introduces the proposed MGPM with residual modification on the basis of a neural network. Section 4 examines prediction performances of the proposed MGPM using two real cases of $\mathrm{CO}_{2}$ emissions. Section 5 discusses the outcomes and presents conclusions.

\section{The GM $(1, N)$ Model}

The GM $(1, N)$ is a grey prediction model with $N$ variables, including a dependent variable (system characteristic), $x_{1}$, and $N-1$ explanatory variables (relevant factors), $x_{2}, x_{3}, \ldots$, $x_{N}$ [5]. Then, an original sequence or a time series $x_{i}^{(0)}=$ $\left(x_{i}^{(0)}(1), x_{i}^{(0)}(2), \ldots, x_{i}^{(0)}(m)\right)$ is associated with $x_{i}$, where $i=1,2, \ldots, N$, and $m$ is the number of samples. $x_{i}^{(0)}$ is usually a sequence taken at successive equally spaced points in time.

Step 1. Present an original and nonnegative sequence $x_{i}^{(0)}$.

Step 2. Perform the accumulated generating operation (AGO).

A new sequence $x_{i}^{(1)}=\left(x_{i}^{(1)}(1), x_{i}^{(1)}(2), \ldots, x_{i}^{(1)}(m)\right)$ can be generated from $x_{i}^{(0)}$ by the AGO as follows:

$$
x_{i}^{(1)}(k)=\sum_{j=1}^{k} x_{0}^{(i)}, \quad k=1,2, \ldots, m .
$$

The AGO can help identify potential hidden regularities in data sequences $[4,43] .\left(x_{i}^{(1)}(1), x_{i}^{(1)}(2), \ldots, x_{i}^{(1)}(m)\right)$ can be approximated by an exponential function, which is a first-order whitening equation,

$$
\frac{\mathrm{d} x_{1}^{(1)}}{\mathrm{d} t}+a x_{1}^{(1)}=\sum_{j=2}^{N} b_{j} x_{j}^{(1)} \text {. }
$$

where $a$ and $b_{j}$ are the development and the driving coefficients, respectively. The time response expression of $\hat{x}_{k}^{(1)}(k)(k=2,3, \ldots, m)$ can be obtained by solving the differential equation with the initial condition $x_{1}^{(1)}(1)=x_{1}^{(0)}(1)$ :

$$
\begin{aligned}
\hat{x}_{1}^{(1)}(k)= & \left(x_{1}^{(0)}(1)-\frac{1}{a} \sum_{j=2}^{N} b_{j} x_{j}^{(1)}(k)\right) e^{-a k} \\
& +\frac{1}{a} \sum_{j=2}^{N} b_{j} x_{j}^{(1)}(k) .
\end{aligned}
$$

Step 3. Determine the development and the driving coefficients.

A grey differential equation of the $\operatorname{GM}(1, N)$ is as follows: 


$$
x_{1}^{(0)}(k)+a z_{1}^{(1)}(k)=\sum_{j=2}^{N} b_{j} x_{j}^{(1)}(k) \quad k=2,3, \ldots, m .
$$

where the background value $z_{1}^{(1)}(k)$ with the generating coefficient $\alpha(0 \leq \alpha \leq 1)$ being usually set to 0.5 is formulated as

$$
\begin{array}{r}
z_{1}^{(1)}(k)=a x_{1}^{(1)}(k)+(1-\alpha) x_{1}^{(1)}(k-1), \\
k=2,3, \ldots, m .
\end{array}
$$

Thus, a linear regression model consisting of grey differential equations is used to estimate $a, b_{2}, \ldots$, and $b_{N}$ through the ordinary least squares (OLS) method:

$$
\left[a, b_{2}, \ldots, b_{N}\right]=\left(B^{T} B\right)^{-1} B^{T} y .
$$

where

$$
\begin{aligned}
& B=\left[\begin{array}{cccc}
-z_{1}^{(1)}(2) & x_{2}^{(1)}(2) & \cdots & x_{N}^{(1)}(2) \\
-z_{1}^{(1)}(3) & x_{2}^{(1)}(3) & \cdots & x_{N}^{(1)}(3) \\
\vdots & \vdots & \ddots & \vdots \\
-z_{1}^{(1)}(m) & x_{2}^{(1)}(m) & \cdots & x_{N}^{(1)}(m)
\end{array}\right], \\
& y=\left[\begin{array}{c}
x_{1}^{(0)}(2) \\
x_{1}^{(0)}(3) \\
\vdots \\
x_{1}^{(0)}(m)
\end{array}\right] .
\end{aligned}
$$

Step 4. Perform the inverse accumulated generating operation (IAGO).

When $x_{i}^{(1)}$ varies slightly, $\hat{x}_{k}^{(0)}$ can be generated by means of the IAGO:

$$
\hat{x}_{1}^{(0)}(k)=\widehat{x}_{1}^{(1)}(k)-\widehat{x}_{1}^{(1)}(k), \quad k=2,3, \ldots, m .
$$

where $\hat{x}_{1}^{(1)}(1)=x_{1}^{(0)}(1)$.

To improve the prediction performance of the traditional GM $(1, N)$, several improved versions of MGPMs have been proposed by deriving new whitening and grey differential equations:

(1) The transformed model of nonlinear GM $(1, N)$ (TNGM $(1, N))[28]$ : the TNGM $(1, N)$ has been applied to forecast Chinese carbon emissions. The whitening equation of the $\operatorname{TNGM}(1, N)$ is defined as

$$
\frac{d x_{1}^{(1)}}{d t}+a x_{1}^{(1)}=\sum_{j=2}^{N} b_{j}\left(x_{j}^{(1)}\right)^{\gamma_{j}} .
$$

The solution of the whitening equation $\hat{x}_{1}^{(0)}(k)$ is thus given by

$$
\begin{aligned}
\widehat{x}_{1}^{(0)}(k)= & \frac{1}{1+0.5 a} \sum_{j=2}^{N} b_{j}\left(x_{j}^{1}(k)\right)^{\gamma_{j}} \\
& -\frac{a}{1+0.5 a} \sum_{j=2}^{N} x_{1}^{(1)}(k-1) .
\end{aligned}
$$

$a, b_{2}, \ldots$, and $b_{N}$ can be further derived by OLS as

$$
\left[a, b_{2}, \ldots, b_{N}\right]=\left(B^{T} B\right)^{-1} B^{T} y .
$$

where

$$
B=\left[\begin{array}{cccc}
-z_{1}^{(1)}(2) & \left(x_{2}^{(1)}(2)\right)^{\gamma_{2}} & \cdots & \left(x_{N}^{(1)}(2)\right)^{\gamma_{N}} \\
-z_{1}^{(1)}(3) & \left(x_{2}^{(1)}(3)\right)^{\gamma_{2}} & \cdots & \left(x_{N}^{(1)}(3)\right)^{\gamma_{N}} \\
\vdots & \vdots & \ddots & \vdots \\
-z_{1}^{(1)}(m) & \left(x_{2}^{(1)}(m)\right)^{\gamma_{2}} & \cdots & \left(x_{N}^{(1)}(m)\right)^{\gamma_{N}}
\end{array}\right] .
$$

Any optimization technique such as a GA can be used to derive the optimal $\gamma_{2}, \gamma_{3}, \ldots$, and $\gamma_{N}$.

(2) The optimization of the GM $(1, N)$ (OGM $(1, N))$ [35]: the grey difference equation of the $\operatorname{OGM}(1, N)$ is defined as

$$
\begin{array}{r}
x_{1}^{(0)}(k)+a z_{1}^{(1)}(k)=\sum_{j=2}^{N} b_{j} x_{j}^{(1)}(k)+h_{1}(k-1)+h_{2}, \\
k=2,3, \ldots, m .
\end{array}
$$

The time response expression of $\hat{x}_{1}^{(1)}(k)$ is thus given by

$$
\begin{aligned}
\hat{x}_{1}^{(1)}(k)= & \sum_{j=2}^{k}\left[\mu_{1} \sum_{m=2}^{N} \mu_{2}^{j-1} b_{m} x_{m}^{(1)}(k-j+1)\right] \\
& +\mu_{2}^{k-1} x_{1}^{(0)}(1)+\sum_{j=0}^{k-2} \mu_{2}^{j}\left[(k-j) \mu_{3}+\mu_{4}\right] .
\end{aligned}
$$

where

$$
\begin{aligned}
& \mu_{1}=\frac{1}{1+0.5 a}, \\
& \mu_{2}=\frac{1-0.5 a}{1+0.5 a}, \\
& \mu_{3}=\frac{h_{1}}{1+0.5 a}, \\
& \mu_{4}=\frac{h_{2}-h_{1}}{1+0.5 a} .
\end{aligned}
$$

$a, b_{2}, \ldots, b_{N}, h_{1}$, and $h_{2}$ can be obtained by OLS as 


$$
\left[a, b_{2}, \ldots, b_{N}, h_{1}, h_{2}\right]=\left(B^{T} B\right)^{-1} B^{T} y
$$

where

$$
B=\left[\begin{array}{cccccc}
-z_{1}^{(1)}(2) & x_{2}^{(1)}(2) & \cdots & x_{N}^{(1)}(2) & 1 & 1 \\
-z_{1}^{(1)}(3) & x_{2}^{(1)}(3) & \cdots & x_{N}^{(1)}(3) & 2 & 1 \\
\vdots & \vdots & \ddots & \vdots & \vdots & \vdots \\
-z_{1}^{(1)}(m) & x_{2}^{(1)}(m) & \cdots & x_{N}^{(1)}(m) & m-1 & 1
\end{array}\right] .
$$

Zeng et al. [25] applied a variant of the $\operatorname{OGM}(1, N)$ to forecast the number of motor vehicles in Beijing.

(3) The MGPM based on trends of driving coefficients (TDVGM $(1, N)$ ) [7]: the development of the TDVGM $(1, N)$ addressed an issue of forecasting Chinese $\mathrm{CO}_{2}$ emissions. This prediction model initially divided data from the first year by data from each year. Then, it predicted trends of driving coefficients by defining the grey differential equation for $b_{j}(j=2,3, \ldots, N)$ as

$$
x_{j}^{(0)}(k)+a_{j} z_{j}^{(1)}(k)=b_{j} .
$$

The time response expression of $\widehat{x}_{j}^{(1)}(k)$ is formulated as

$$
\widehat{x}_{j}^{(1)}(k)=\left(1-\frac{b_{j}}{a_{j}}\right) e^{-a j(k-1)}+\frac{b_{j}}{a_{j}}, \quad k=2,3, \ldots, m .
$$

$a_{j}$ and $b_{j}$ were obtained by OLS as

$$
\left[a_{j}, b_{j}\right]=\left(B^{T} B\right)^{-1} B^{T} y
$$

where

$$
B=\left[\begin{array}{cc}
-z_{1}^{(1)}(2), & 1 \\
-z_{1}^{(1)}(3), & 1 \\
\vdots & \vdots \\
-z_{1}^{(1)}(m), & 1 .
\end{array}\right]
$$

Then, a time response expression of $\hat{x}_{1}^{(1)}(k)$ can be derived as

$$
\begin{aligned}
\widehat{x}_{1}^{(1)}(k)= & c e^{-a k}+\left(\sum_{j=2}^{N} \frac{d_{j}\left(\left(1-b_{j}\right) / a_{j}\right)}{a-a_{j}} e^{-a_{j}(k-1)}\right) e^{-a k} \\
& +\sum_{j=2}^{N} \frac{d_{j} b_{j}}{a a_{j}}
\end{aligned}
$$

where

$$
\begin{aligned}
& c=c e^{-a k}+\frac{\sum_{k=1}^{m} e^{-a k}\left(x_{1}^{(0)}(k)-\sum_{j=2}^{N} d_{j}\left(1-b_{j} / a_{j}\right) / a-a_{j}\left(1-e^{a_{j}}\right) e^{-a_{j}(k-1)}\right)}{\sum_{k=1}^{n}\left(1-e^{a}\right)^{2} e^{-2 a k}} . \\
& \quad \xi j k=\frac{\Delta_{\min }+\rho \Delta_{\max }}{\Delta_{j k}+\rho \Delta_{\max }},
\end{aligned}
$$

\section{The Proposed Multivariate Grey Prediction Model}

For the proposed MGPM, GRA was first used to keep explanatory variables that are more relevant to $\mathrm{CO}_{2}$ emissions. Then, the proposed MGPM can be constructed by GAs. Subsequently, a residual GM $(1,1)$ model can be embedded into the proposed MGPM by establishing a functional-link net to adjust $\widehat{x}_{1}^{(0)}(k)$.

3.1. Feature Selection by Grey Relational Analysis. In contrast to statistical correlation analysis that measures the relationship between any two random variables, GRA can effectively measure the relationships between one reference sequence and the other comparative sequences by viewing the reference sequence as the desired goal [44]. The grey relational coefficient, $\xi_{j k}$, for the time period $k(1 \leq j \leq N-1$, $1 \leq k \leq m)$ is addressed by the discriminative coefficient $\rho$ $(0 \leq \rho \leq 1)$ to indicate the relationship between $x_{j}^{(0)}(k)$ and $x_{1}^{(0)}(k)$ : where $\rho$ is usually specified as 0.5 and

$$
\begin{aligned}
\Delta \min & =\min _{j=1, \ldots, N-1} \min _{k=1, \ldots, m}\left|x_{1}^{(0)}(k)-x_{j}^{(0)}(k)\right|, \\
\Delta \min & =\max _{j=1, \ldots, N-1} \max _{k=1, \ldots, m}\left|x_{1}^{(0)}(k)-x_{j}^{(0)}(k)\right|, \\
\Delta j k & =\left|x_{1}^{(0)}(k)-x_{j}^{(0)}(k)\right| .
\end{aligned}
$$

It is seen that $\xi_{j k}$ lies in $[0,1]$ and approaches 1 if $\Delta_{j k}$ approaches $\Delta_{\min }$.

To measure the degree of proximity between $x_{j}$ and $x_{1}$, the grey relational grade (GRG) $\gamma_{j}$ can be calculated as follows:

$$
\gamma_{j}=\frac{1}{m} \sum_{k=1}^{m} \xi_{i k}
$$

where $\gamma_{j}$ ranges from 0 to 1 . The greater the value of $\gamma_{i}$ is, the more relevant $x_{j}$ is to $x_{1}$. In other words, to construct the proposed model, $x_{j}$ can be retained for constructing the 
proposed MGPM when $\gamma_{j}$ surpasses a threshold value $\lambda$; otherwise, $x_{j}$ can no longer be considered for the proposed MGPM. That means, for equation (3), both $b_{j}$ and $x_{j}^{(1)}(k+$ 1) associated with $x_{j}$ can be retained when $\gamma_{j}$ is above $\lambda$; otherwise, they can be removed directly. However, $\lambda$ may not be prespecified easily beforehand.

3.2. Construction of Multivariate Grey Prediction Models Using Genetic Algorithms. This study aims to find the optimal solution to construct the proposed MGPM with high prediction accuracy. This problem can be thus formulated as the following single objective optimization problem by minimizing the mean absolute percentage error (MAPE):

$$
\operatorname{Minimize} \frac{1}{m-1} \sum_{k=2}^{m} \frac{\left|x_{1}^{(0)}(k)-\widehat{x}_{1}^{(0)}(k)\right|}{x_{1}^{(0)}(k)} .
$$

Instead of OLS, a real-valued GA using MAPE as its fitness function can be developed to automatically determine the optimal values of development coefficient $(a)$, the driving coefficients $\left(b_{2}, b_{3}, \ldots, b_{N}\right)$, and the cut value $(\lambda)$.

For the GA, a set of strings making up a population is generated initially. All parameters for each string are randomly generated as real numbers. Using the Genetic Algorithm and the Direct Search Toolbox in MATLAB, a realvalued GA is easily developed to automatically determine all parameters. The best chromosome with the maximum fitness value of all successive generations is the desired solution for examining the generalizability of the proposed MGPM.

3.3. Residual Modification Using a Functional-Link Net. $\widehat{x}_{1}^{(0)}(k)$ produced by the proposed MGPM can be further adjusted by residual modification to improve prediction accuracy. Let $\varepsilon_{1}^{(0)}=\left(\varepsilon_{1}^{(0)}(2), \varepsilon_{1}^{(0)}(3), \ldots, \varepsilon_{1}^{(0)}(m)\right)$ denote the sequence of absolute residual values, where

$$
\varepsilon_{1}^{(0)}(k)=\left|\varepsilon_{1}^{(0)}(k)-\widehat{\varepsilon}_{1}^{(0)}\right|, \quad k=2,3, \ldots, m .
$$

Because no dependent variables are considered for $\varepsilon_{1}$, a residual $\operatorname{GM}(1,1)$ model can be established for $\varepsilon_{1}^{(0)}$, where the predicted value of $\varepsilon_{1}^{(0)}(k)$ is

$$
\widehat{\varepsilon}_{1}^{(0)}(k)=\left(1-e^{a_{e}}\right)\left(\varepsilon_{1}^{(0)}(2)-\frac{b_{\varepsilon}}{a_{\varepsilon}}\right) e^{-a e(k-1)}, \quad k=3,4, \ldots, m,
$$

where $a_{\varepsilon}$ and $b_{\varepsilon}$ are the developing coefficient and the control variable, respectively. $\widehat{\varepsilon}_{1}^{(1)}(2)=\varepsilon_{1}^{(0)}(2)$. The problem of constructing a residual GM $(1,1)$ model can be formulated as the following single objective optimization problem by minimizing the MAPE:

$$
\operatorname{Minimize} \sum_{k=3}^{m} \frac{\left|\varepsilon_{1}^{(0)}(k)-\widehat{\varepsilon}_{1}^{(0)}(k)\right|}{\varepsilon_{1}^{(0)}(k)} .
$$

MAPE can be used as the fitness function of a real-valued GA that is used to determine optimal $a_{\varepsilon}$ and $b_{\varepsilon}$ instead of OLS.
Then, following a mechanism of residual modification recommended by Wang and $\mathrm{Hu}$ [41], the final predicted value $\hat{x}_{k}^{(0)}$ of the proposed MGPM is produced by means of $\widehat{x}_{1}^{(0)}(k)$ :

$$
\widehat{x}_{1}^{(0)}(k)=\widehat{x}_{1}^{(0)}(k)+3 y_{k} \widehat{\varepsilon}_{1}^{(0)}(k), \quad k=2,3, \ldots, n,
$$

where $y_{k}$ ranges from -1 to 1 and can be computed by presenting $\left(t_{k}, \sin \left(\pi t_{k}\right), \cos \left(\pi t_{k}\right), \sin \left(2 \pi t_{k}\right)\right.$, and $\left.\cos \left(2 \pi t_{k}\right)\right)$ to a single-layer perceptron, namely, the functional-link net, with effective function approximation capability [45-48]:

$$
\begin{aligned}
y_{k}= & \tanh \left(w_{1} t_{k}+w_{2} \sin \left(\pi t_{k}\right)+w_{3} \cos \left(\pi t_{k}\right)\right. \\
& \left.+w_{4} \sin \left(2 \pi t_{k}\right)+w_{5} \cos \left(2 \pi t_{k}\right)+\theta\right),
\end{aligned}
$$

where $\tan h$ represents a hyperbolic tangent function, and $w_{1}, \ldots, w_{5}$ are connection weights. This means that the amount of adjusting $\widehat{x}_{k}^{(0)}$ could be as much as $3 \widehat{\varepsilon}_{k_{0}}^{(0)}$ if $y_{k}=1$. In contrast, the adjustable amount can be $-3 \widehat{\varepsilon}_{k}^{(0)}$ if $y_{k}=-1$.

\section{Empirical Results}

Empirical studies were conducted using real datasets to compare the $\mathrm{CO}_{2}$ emission forecasting ability of the proposed MGPM with the other MGPMs considered. As mentioned above, urban population (UP), GDP, and energy consumption have a dominant influence on $\mathrm{CO}_{2}$ emissions. In addition to the traditional GM $(1, N)$, the Autoregressive Integrated Moving Average model (ARIMA) and the aforementioned improved MGPMs with comprehensible distinctive features were considered.

4.1. Case I. Statistics from the IEA [3] revealed that, in 2015, the total amount of $\mathrm{CO}_{2}$ emissions in China was 9,040 million tons, reaching the highest level worldwide. To devise energy plans that would effectively reduce $\mathrm{CO}_{2}$ emissions while promoting green economic growth, the ability to predict $\mathrm{CO}_{2}$ emissions has played a very significant role in China. Therefore, we were intrigued to examine the prediction performance of MGPMs that consider $\mathrm{CO}_{2}$ emissions in China. The data on urban population (million persons) and GDP (million USD dollars) were collected from the World Bank (http://data.worldbank.org.cn), and energy consumption (million tons of oil equivalent) and $\mathrm{CO}_{2}$ emissions (million tons) were collected from the IEA (http://www.iea.org).

As shown in Table 1, the historical annual data were collected from 2005 to 2015, data from 2005 to 2012 were used for the model-fitting, and data from 2013 to 2015 were used for ex-post testing. Results shown in Table 2 are summarized as follows:

(1) The MAPE of the traditional GM $(1, N)$, the TNGM $(1, N)$, the $\operatorname{OGM}(1, N)$, the TDVGM $(1, N)$, the ARIMA, and the proposed MGPM for model-fitting were $3.34 \%, 0.81 \%, 0.02 \%, 3.14 \%, 1.98 \%$, and $1.71 \%$, respectively. The OGM $(1, N)$ thus demonstrated its superiority in model-fitting. 
Table 1: Annual carbon dioxide emissions with GDP, UP, and EC in China.

\begin{tabular}{lcccc}
\hline Year & GDP & UP & EC & CO $_{2}$ \\
\hline 2005 & 2285966 & 554.37 & 1184.158 & \\
2006 & 2752132 & 575.12 & 1273.683 & \\
2007 & 3552182 & 595.67 & 1368.733 & \\
2008 & 4598206 & 616.48 & 1417.323 & \\
2009 & 5109954 & 637.41 & 1480.672 & \\
2010 & 6100620 & 658.50 & 1578.852 & 70258.96 \\
2011 & 7572554 & 679.77 & 1692.063 & 7706.65 \\
2012 & 8560547 & 700.86 & 1747.103 & 8465.64 \\
2013 & 9607224 & 721.69 & 1816.852 & 8620.58 \\
2014 & 10482372 & 742.30 & 1868.17 & 8995.79 \\
2015 & 11064666 & 762.59 & 1905.679 & 9036.47 \\
\hline
\end{tabular}

TABle 2: MAPE (\%) obtained by different MGPMs for carbon dioxide emissions in China.

\begin{tabular}{|c|c|c|c|c|c|c|c|c|c|c|c|c|c|}
\hline \multirow[t]{2}{*}{ Year } & \multirow[t]{2}{*}{ Actual } & \multicolumn{2}{|c|}{ GM $(1, N)$} & \multicolumn{2}{|c|}{$\operatorname{TNGM}(1, N)$} & \multicolumn{2}{|c|}{ OGM $(1, N)$} & \multicolumn{2}{|c|}{$\operatorname{TDVGM}(1, N)$} & \multicolumn{2}{|c|}{ ARIMA } & \multicolumn{2}{|c|}{$\begin{array}{l}\text { The proposed } \\
\text { MGPM }\end{array}$} \\
\hline & & Predicted & $\mathrm{APE}$ & Predicted & APE & Predicted & APE & Predicted & APE & Predicted & APE & Predicted & APE \\
\hline 2005 & 57.7 & 5357.7 & 0.00 & 5357.7 & 0.00 & 5357.7 & 0.00 & 5435.9 & 1.32 & 5357.71 & 0.00 & 5357.7 & 0.00 \\
\hline 2006 & 12.0 & 5007.9 & 15.29 & 5869.3 & 0.72 & 5912.1 & 0.00 & 5786.9 & 2.12 & 5916.89 & 0.08 & 5919.4 & .13 \\
\hline 2007 & 68.3 & 6941.3 & 7.31 & 6478.3 & 0.15 & 6467.7 & 0.01 & 6160.3 & 4.76 & 6437.24 & 0.48 & 6850.5 & 5.91 \\
\hline 2008 & 08.1 & 6753.8 & 2.20 & 6495.6 & 1.70 & 6609.7 & 0.02 & 6551.9 & 0.85 & 6919.37 & 4.71 & 6739.0 & 1.98 \\
\hline 2009 & 25.8 & 7003.8 & 0.31 & 7083.0 & 0.81 & 7023.3 & 0.04 & 6962.3 & 0.90 & 7366.09 & 4.84 & 7026.6 & 0.01 \\
\hline 2010 & 7706.7 & 7651.5 & 0.72 & 7703.0 & 0.05 & 7709.3 & 0.03 & 7390.9 & 4.10 & 7779.98 & 0.95 & 7556.9 & 1.94 \\
\hline 2011 & 465.6 & 8429.1 & 0.43 & 8255.0 & 2.49 & 8464.0 & 0.02 & 7838.3 & 7.41 & 8163.48 & 3.57 & 8186.0 & 3.30 \\
\hline 2012 & 8620.6 & 8660.7 & 0.47 & 8665.3 & 0.52 & 8621.1 & 0.01 & 8303.9 & 3.67 & 8518.80 & 1.18 & 8582.9 & 0.44 \\
\hline MAPE & - & - & 3.34 & - & 0.81 & - & 0.02 & - & 3.14 & - & 1.98 & - & 1.71 \\
\hline 2013 & 8995.8 & 9039.7 & 0.49 & 9011.6 & 0.18 & 9080.8 & 0.94 & 8788.2 & 2.31 & 8848.02 & 1.64 & 8990.6 & 0.06 \\
\hline 2014 & 9036.5 & 9248.2 & 2.34 & 9270.5 & 2.59 & 9282.2 & 2.72 & 9290.3 & 2.81 & 9153.05 & 1.29 & 9242.6 & 2.28 \\
\hline 2015 & 9040.7 & 9342.8 & 3.34 & 9452.7 & 4.56 & 9419.1 & 4.19 & 9809.9 & 8.51 & 9435.68 & 4.37 & 9335.2 & 3.26 \\
\hline MAPE & - & - & 2.06 & - & 2.44 & - & 2.62 & - & 4.54 & - & 2.43 & - & 1.87 \\
\hline
\end{tabular}

(2) For ex-post testing, the MAPE of the traditional GM $(1, N)$, the TNGM $(1, N)$, the OGM $(1, N)$, the TDVGM $(1, N)$, the ARIMA, and the proposed MGPM were $2.06 \%, 2.44 \%, 2.62 \%, 4.54 \%, 2.43 \%$, and $1.87 \%$, respectively.

(3) Although the proposed MGPM is inferior to the TNGM $(1, N)$ and the OGM $(1, N)$ for model-fitting, it is superior to other MGPMs considered for ex-post testing.

It should be noted that, when evaluating a prediction model, more emphasis should be placed on generalization rather than on model-fitting [49]. Figure 1 also demonstrates the superiority of the generalization ability of the proposed MGPM over the other prediction models considered.

4.2. Case II. From statistics reported by the IEA [47], the total and average amounts of $\mathrm{CO}_{2}$ emissions in Taiwan in 2015 were the 21st and the 19th highest in the world, respectively. This means that Taiwan still has room to reduce $\mathrm{CO}_{2}$ emissions. The second real case involved the historical annual $\mathrm{CO}_{2}$ emission data collected in Taiwan from 2005 to 2015. The data on urban population (million persons) and GDP (million USD dollars) were collected from the United
Nations Conference on Trade and Development (UNCTAD) (http://unctad.org/en/Pages/statistics.aspx), and energy consumption (million tons of oil equivalent) and $\mathrm{CO}_{2}$ emissions (million tons) were collected from the IEA.

As shown in Table 3, data collected from 2005 to 2012 were used for the model-fitting, and data from 2013 to 2015 were used for ex-post testing. The results obtained from the different prediction models are shown in Table 4 . The results are summarized as follows:

(1) The MAPE of the traditional GM $(1, N)$, the TNGM $(1, N)$, the $\operatorname{OGM}(1, N)$, the $\operatorname{TDVGM}(1, N)$, the ARIMA, and the proposed MGPM for model-fitting were $3.35 \%, 0.23 \%, 0.21 \%, 2.59 \%, 2.10 \%$, and $1.86 \%$, respectively. The TNGM $(1, N)$ and the $\operatorname{OGM}(1, \mathrm{~N})$ demonstrated their superiority in model-fitting.

(2) The MAPE of the traditional GM $(1, N)$, the TNGM $(1, N)$, the OGM $(1, N)$, the $\operatorname{TDVGM}(1, N)$, the ARIMA, and the proposed MGPM for ex-post testing were $1.28 \%, 4.75 \%, 8.43 \%, 4.54 \%, 0.86 \%$, and $1.09 \%$, respectively. The proposed MGPM is slightly inferior to the TDVGM $(1, N)$.

(3) Although the proposed MGPM is inferior to the $\operatorname{TNGM}(1, N)$ and the OGM $(1, N)$ for model-fitting, it is superior to those two MGPMs for ex-post testing. 


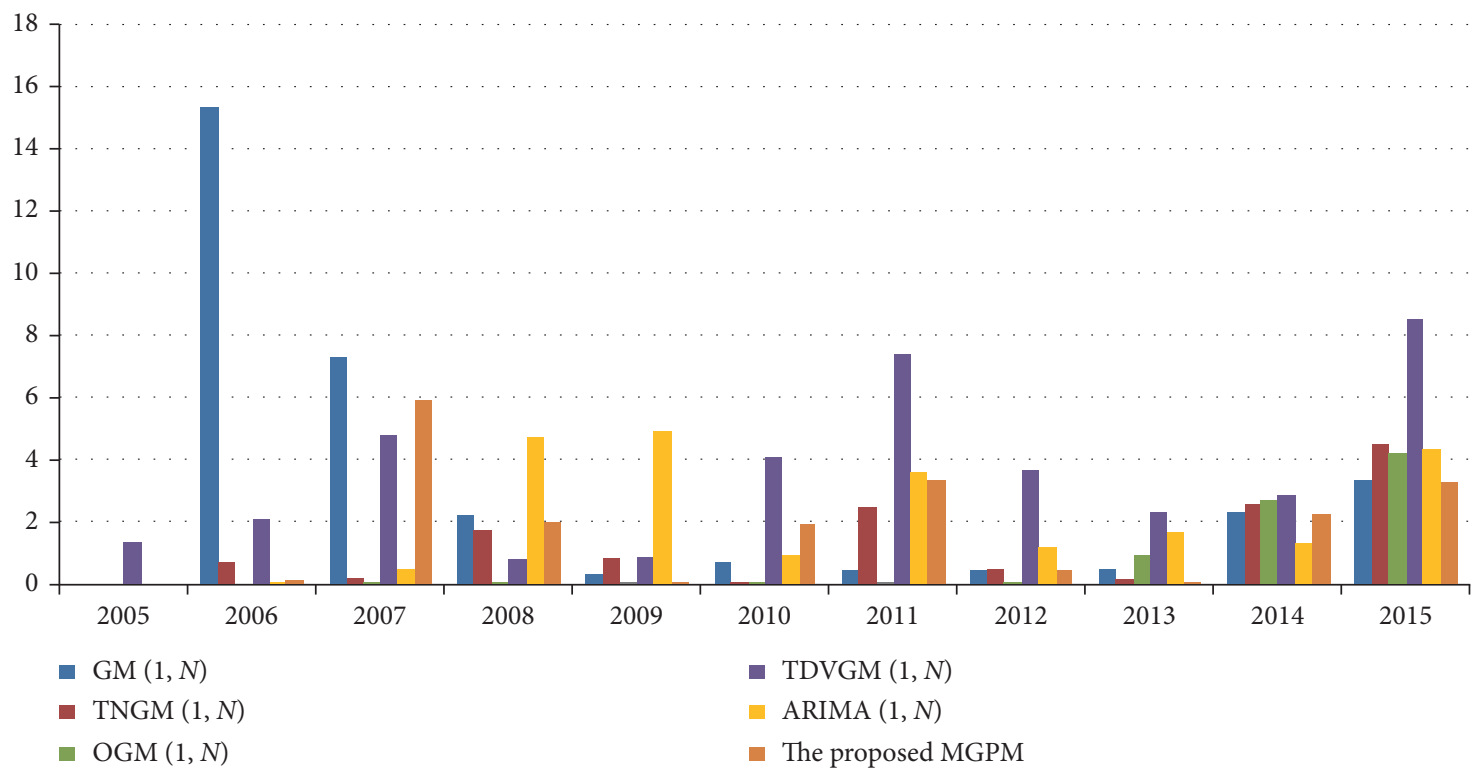

Figure 1: Comparisons between different MGPMs for carbon dioxide emissions prediction in China.

TABLE 3: Annual carbon dioxide emissions with GDP, UP, and EC in Taiwan.

\begin{tabular}{ccccc}
\hline Year & GDP & UP & EC & CO $_{2}$ \\
\hline 2005 & 375787 & 22.603 & 60.437 & 253.64 \\
2006 & 388547 & 22.725 & 61.276 & 260.86 \\
2007 & 408221 & 22.833 & 65.511 & 263.94 \\
2008 & 417038 & 22.929 & 63.393 & 2.75 \\
2009 & 392106 & 23.017 & 62.892 & 239.68 \\
2010 & 446141 & 23.102 & 67.855 & 256.22 \\
2011 & 485671 & 23.185 & 65.405 & 254.7 \\
2012 & 495919 & 23.264 & 65.289 & 246.55 \\
2013 & 511599 & 23.34 & 67.808 & 247.59 \\
2014 & 530515 & 23.414 & 68.014 & 249.66 \\
2015 & 525236 & 23.486 & 68.566 & 249.38 \\
\hline
\end{tabular}

TABLE 4: MAPE (\%) obtained by different MGPMs for carbon dioxide emissions in Taiwan.

\begin{tabular}{|c|c|c|c|c|c|c|c|c|c|c|c|c|c|}
\hline \multirow[t]{2}{*}{ Year } & \multirow[t]{2}{*}{ Actual } & \multicolumn{2}{|c|}{$\mathrm{GM}(1, N)$} & \multicolumn{2}{|c|}{$\operatorname{TNGM}(1, N)$} & \multicolumn{2}{|c|}{ OGM $(1, N)$} & \multicolumn{2}{|c|}{ TDVGM $(1, N)$} & \multicolumn{2}{|c|}{ ARIMA } & \multicolumn{2}{|c|}{$\begin{array}{l}\text { The proposed } \\
\text { MGPM }\end{array}$} \\
\hline & & Predicted & $\mathrm{APE}$ & Predicted & APE & Predicted & APE & Predicted & APE & Predicted & APE & Predicted & APE \\
\hline 2005 & 253.64 & 253.64 & 0.00 & 253.64 & 0.00 & 253.64 & 0.00 & 250.18 & 1.36 & 253.64 & 0.00 & 253.64 & 0.00 \\
\hline 2006 & 260.86 & 220.24 & 15.57 & 260.33 & 0.20 & 260.38 & 0.18 & 250.79 & 3.86 & 255.91 & 1.90 & 261.36 & 0.19 \\
\hline 2007 & 263.94 & 270.58 & 2.52 & 264.40 & 0.17 & 264.29 & 0.13 & 251.06 & 4.88 & 251.58 & 4.68 & 258.87 & 1.92 \\
\hline 2008 & 252.75 & 253.11 & 0.14 & 253.18 & 0.17 & 252.48 & 0.11 & 251.24 & 0.60 & 252.39 & 0.14 & 249.56 & 1.26 \\
\hline 2009 & 239.68 & 252.71 & 5.44 & 239.68 & 0.00 & 239.66 & 0.01 & 251.36 & 4.87 & 252.24 & 5.24 & 233.84 & 2.44 \\
\hline 2010 & 256.22 & 256.63 & 0.16 & 255.82 & 0.16 & 256.81 & 0.23 & 251.43 & 1.87 & 252.26 & 1.54 & 250.40 & 2.27 \\
\hline 2011 & 254.70 & 249.01 & 2.23 & 252.97 & 0.68 & 252.88 & 0.71 & 251.43 & 1.28 & 252.26 & 0.96 & 241.07 & 5.35 \\
\hline 2012 & 246.55 & 248.41 & 0.75 & 247.71 & 0.47 & 247.24 & 0.28 & 251.37 & 1.95 & 252.26 & 2.32 & 242.89 & 1.48 \\
\hline MAPE & - & - & 3.35 & - & 0.23 & - & 0.21 & - & 2.59 & - & 2.10 & - & 1.86 \\
\hline 2013 & 253.64 & 251.97 & 1.77 & 254.77 & 2.90 & 257.98 & 4.20 & 251.24 & 1.47 & 252.26 & 1.89 & 249.91 & 0.94 \\
\hline 2014 & 260.86 & 251.07 & 0.56 & 260.44 & 4.32 & 268.40 & 7.51 & 251.04 & 0.55 & 252.26 & 1.04 & 251.72 & 0.83 \\
\hline 2015 & 263.94 & 253.13 & 1.50 & 266.89 & 7.02 & 283.29 & 13.60 & 250.77 & 0.56 & 252.26 & 1.15 & 253.15 & 1.51 \\
\hline MAPE & - & - & 1.28 & - & 4.75 & - & 8.43 & - & 0.86 & - & 1.36 & - & 1.09 \\
\hline
\end{tabular}




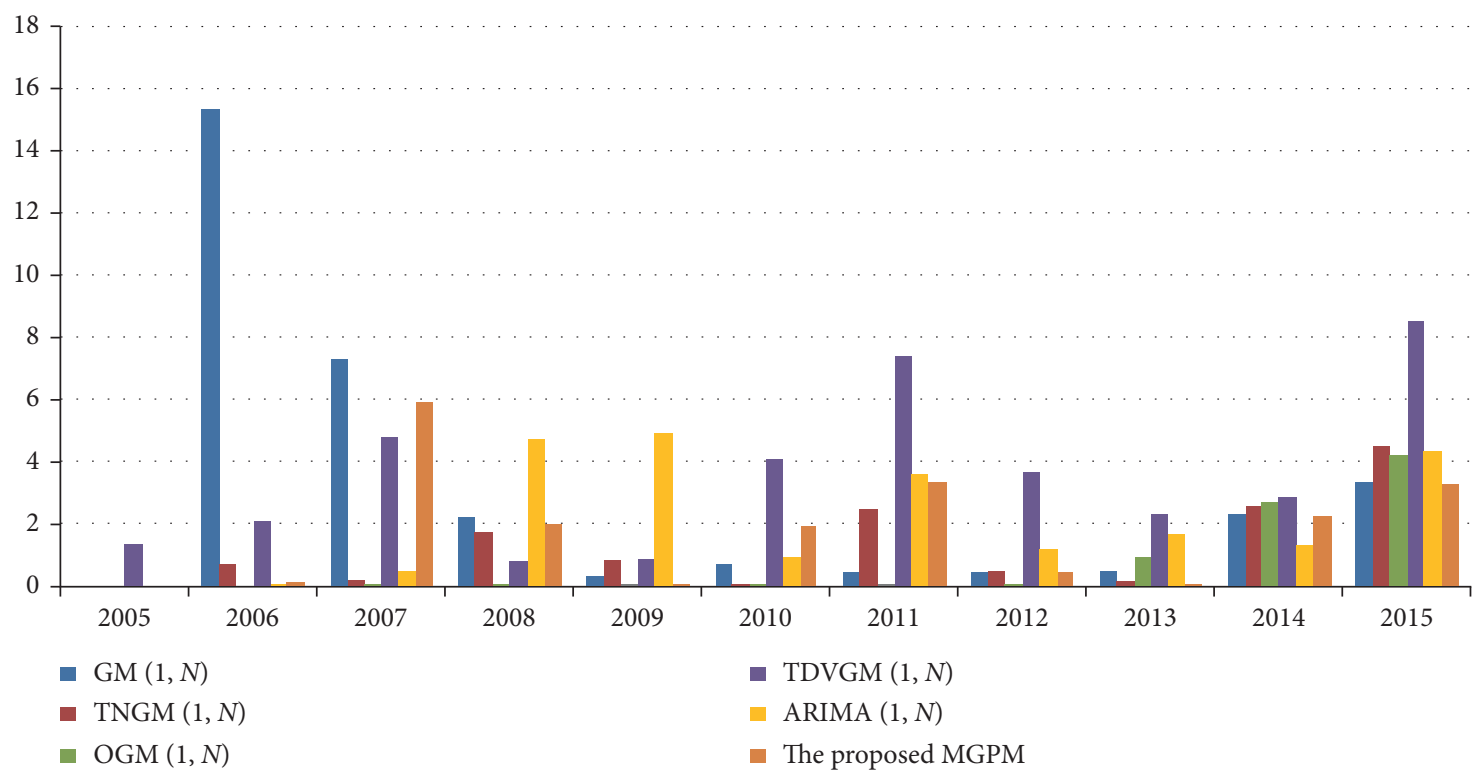

FIgURe 2: Comparisons between different MGPMs for carbon dioxide emissions prediction in Taiwan.

In Figure 2, we can see that the proposed MGPM performs well compared with other prediction models considered.

\section{Discussion}

What this study focuses on is the forecast rather than the projection. Compared with the projection, the forecast places value on estimating the amount of $\mathrm{CO}_{2}$ emissions based on an established model such as a MGPM for which MAPE is a useful metric for measuring the prediction performance [50]. The projection can answer "what-if" kind of questions to extrapolate the development trend. In other words, it is concerned about what would happen to $\mathrm{CO}_{2}$ emission based on some future scenarios, for instance, how $\mathrm{CO}_{2}$ is produced and how it is influenced by what kind of factors.

As mentioned above, the forecast of $\mathrm{CO}_{2}$ emissions can be regarded as a grey system problem. Furthermore, $\mathrm{CO}_{2}$ emission data might well not conform to statistical assumptions. Therefore, it is reasonable to apply MGPMs to forecast the amount of $\mathrm{CO}_{2}$ emissions. Compared with the other MGPMs, feature selection and residual modification are taken into account in the proposed MGPM to improve prediction accuracy. In particular, GRA and a functionallink net are employed to implement feature selection and residual modification, respectively. The empirical results reveal that feature selection and residual modification can boost the prediction performance of the proposed MGPM. It is noted that although the $\operatorname{OGM}(1, N)$ variant [25] also applied GRA to filter out irrelevant features, the cut value $(\lambda)$ was arbitrarily assigned by a prespecified value. However, $\lambda$ can be optimized by the GA to improve the prediction accuracy of the proposed MGPM.

With real-world datasets, from Tables 2 and 4, we can see that the generalization ability of the proposed MGPM for $\mathrm{CO}_{2}$ emissions was quite encouraging. The outcomes verified that the results obtained by the proposed MGPM are comparable to other prediction models considered. It is interesting to note that the TNGM $(1, N)$ and the OGM $(1$, $N)$ are superior to the traditional GM $(1, N)$ and the proposed MGPM for model-fitting but inferior for ex-post testing. In other words, both the TNGM $(1, N)$ and the OGM $(1, N)$ appear to be overfitting. Experimental results show that the fitting and generalization abilities of the proposed MGPM are superior to the traditional GM $(1, N)$. Thus, the prediction ability of the traditional GM $(1, N)$ could be effectively improved by feature selection and residual modification indeed.

\section{Conclusions}

Undoubtedly, the reduction of greenhouse gas emissions is critical to environmental protection. For many countries, $\mathrm{CO}_{2}$ is mainly produced from fuel combustion, which forms the majority of greenhouse gases. How to reduce the impact that energy consumption and economic growth have on $\mathrm{CO}_{2}$ emissions has gained increasing global attention. Revelations from the IEA [3] showed that, along with global economic growth, global $\mathrm{CO}_{2}$ emissions plateaued from 2014 to 2016 due to the growth of renewable electricity, the replacement of coal with natural gas, and changes to the economic structure. There appeared to be a decoupling of economic growth and environmental degradation. However, this does not mean that $\mathrm{CO}_{2}$ emissions have reached a summit. To continuously inhibit carbon emissions and remain competitive, it is necessary for the authorities to make use of prediction models on carbon emissions, set up comprehensive policies on the development of new energy technologies, and increase demand for renewable energies (e.g., solar, wind, and natural gas) and environmental protection.

In addition to $\mathrm{CO}_{2}$ emissions, there are several multivariate prediction problems, such as energy demand 
forecasting, which need to be resolved. In fact, energy demand prediction has become increasingly important when devising development plans for a country, particularly for developing countries [51]. Meanwhile, energy demand forecasting can be regarded as a grey system problem [52] because a few factors, such as income and population, have an influence on energy demand, but how exactly these factors affect energy demand is not clear. On the basis of the conspicuous forecasting performance of the proposed MGPM for $\mathrm{CO}_{2}$ emissions, it would be interesting to explore its applicability to energy demand forecasting.

\section{Data Availability}

Statistics used in this paper are from the IEA (International Energy Agency).

\section{Conflicts of Interest}

The authors declare that there are no conflicts of interest.

\section{Acknowledgments}

This research was supported by the Ministry of Science and Technology, Taiwan, under Grant MOST 108-2410-H-033038-MY2, the National Natural Science Foundation of China (no. 71772106), Humanities and Social Sciences Foundation of the Ministry of Education of China (no. 17YJCZH198), Shandong Provincial Natural Science Foundation, China (No. ZR2017MG012).

\section{References}

[1] P. Nema, S. Nema, and P. Roy, "An overview of global climate changing in current scenario and mitigation action," Renewable and Sustainable Energy Reviews, vol. 16, no. 4, pp. 2329-2336, 2012.

[2] W. Wu, X. Ma, Y. Zhang, and Y. Wang, "A novel conformable fractional non-homogeneous grey model for forecasting carbon dioxide emissions of BRICS countries," Science of The Total Environmen, vol. 707, p. 13544, 2020.

[3] International Energy Agency, Key World Energy Statistics 2017, IEA/OECD, Paris, France, 2019.

[4] S. Liu and Y. Lin, Grey Information: Theory and Practical Applications, Springer, Berlin, Germany, 2010.

[5] S. Liu, Y. Yang, and J. Forrest, Grey Data Analysis: Methods, Models and Applications, Springer, Berlin, Germany, 2017.

[6] J. L. Deng, "Control problems of grey systems," Systems and Control Letters, vol. 1, no. 5, pp. 288-294, 1982.

[7] S. Ding, Y.-G. Dang, X.-M. Li, J.-J. Wang, and K. Zhao, "Forecasting Chinese CO2 emissions from fuel combustion using a novel grey multivariable model," Journal of Cleaner Production, vol. 162, pp. 1527-1538, 2017.

[8] L. Wu, S. Liu, D. Liu, Z. Fang, and H. Xu, "Modelling and forecasting CO 2 emissions in the BRICS (Brazil, Russia, India, China, and South Africa) countries using a novel multivariable grey model," Energy, vol. 79, pp. 489-495, 2015.

[9] O. Burcu, "The nexus between carbon emissions, energy consumption and economic growth in Middle East countries: a panel data analysis," Energy Policy, vol. 62, pp. 1138-1147, 2013.
[10] P. A. González and J. M. Zamarreño, "Prediction of hourly energy consumption in buildings based on a feedback artificial neural network," Energy and Buildings, vol. 37, no. 6, pp. 595-601, 2005.

[11] P. Lauret, E. Fock, R. N. Randrianarivony, and J.-F. ManicomRamsamy, "Bayesian neural network approach to short time load forecasting," Energy Conversion and Management, vol. 49, no. 5, pp. 1156-1166, 2008.

[12] C. Xia, J. Wang, and K. McMenemy, "Short, medium and long term load forecasting model and virtual load forecaster based on radial basis function neural networks," International Journal of Electrical Power \& Energy Systems, vol. 32, no. 7, pp. 743-750, 2010.

[13] W. Sun and M. Liu, "Prediction and analysis of the three major industries and residential consumption $\mathrm{CO} 2$ emissions based on least squares support vector machine in China," Journal of Cleaner Production, vol. 122, pp. 144-153, 2016.

[14] O. Kaynar, I. Yilmaz, and F. Demirkoparan, "Forecasting of natural gas consumption with neural network and neuro fuzzy system," Energy Education Science and Technology Part A: Energy Science and Research, vol. 26, pp. 221-238, 2011.

[15] R. Li, X. Chen, T. Balezentis, D. Streimikiene, and Z. Niu, "Multi-step least squares support vector machine modeling approach for forecasting short-term electricity demand with application," Neural Computing and Applications, 2020.

[16] M. Meng and D. Niu, "Modeling $\mathrm{CO}_{2}$ emissions from fossil fuel combustion using the logistic equation," Energy, vol. 36, no. 5, pp. 3355-3359, 2011.

[17] A. Azadeh, M. Khakestani, and M. Saberi, "A flexible fuzzy regression algorithm for forecasting oil consumption estimation," Energy Policy, vol. 37, no. 12, pp. 5567-5579, 2009.

[18] Q. Yan, W. Zhang, J. Yuan, T. Balezentis, and Y. Zhang, "How much electricity will be consumed in 2020 under the new normal economy in China?" Transformations in Business \& Economics, vol. 18, no. 2, pp. 88-102, 2019.

[19] V. Ş. Ediger and S. Akar, "ARIMA forecasting of primary energy demand by fuel in Turkey," Energy Policy, vol. 35, no. 3, pp. 1701-1708, 2007.

[20] S. Tutun, C.-A. Chou, and E. Canıyılmaz, "A new forecasting framework for volatile behavior in net electricity consumption: a case study in Turkey," Energy, vol. 93, pp. 2406-2422, 2015.

[21] C.-H. Wang and L.-C. Hsu, "Using genetic algorithms grey theory to forecast high technology industrial output," Applied Mathematics and Computation, vol. 195, no. 1, pp. 256-263, 2008.

[22] K. L. Wen, Grey Systems Modeling and Prediction, Yang's Scientific Research Institute, Tucson, AZ, USA, 2004.

[23] H. Duan, D. Wang, X. Pang, Y. Liu, and S. Zeng, "A novel forecasting approach based on multi-kernel nonlinear multivariable grey model: a case report," Journal of Cleaner Production, vol. 260, 2020.

[24] M. Guo, J. Lan, J. Li, Z. Lin, and X. Sun, "Traffic flow data recovery algorithm based on gray residual GM $(1, N)$ model," Journal of Transportation Systems Engineering and Information Technology, vol. 12, no. 1, pp. 42-47, 2012.

[25] B. Zeng, C. Luo, S. Liu, and C. Li, "A novel multi-variable grey forecasting model and its application in forecasting the amount of motor vehicles in Beijing," Computers \& Industrial Engineering, vol. 101, pp. 479-489, 2016.

[26] Z.-X. Wang, "A GM $(1, N)$-based economic cybernetics model for the high-tech industries in China," Kybernetes, vol. 43, no. 5, pp. 672-685, 2014. 
[27] L. L. Pei, W. M. Chen, J. H. Bai, and Z. X. Wang, "The improved GM $(1, N)$ models with optimal background values: a case study of Chinese high-tech industry," The Journal of Grey System, vol. 27, no. 3, pp. 223-233, 2015.

[28] Z.-X. Wang and D.-J. Ye, "Forecasting Chinese carbon emissions from fossil energy consumption using non-linear grey multivariable models," Journal of Cleaner Production, vol. 142, pp. 600-612, 2017.

[29] Z. X. Wang and P. Hao, "An improved grey multivariable model for predicting industrial energy consumption in China," Applied Mathematical Modelling, vol. 40, no. 11-12, pp. 5745-5758, 2016.

[30] L. C. Hsu, "Forecasting the output of integrated circuit industry using genetic algorithm based multivariable grey optimization models," Expert Systems with Applications, vol. 36, no. 2, pp. 7898-7903, 2009.

[31] L.-C. Hsu and C.-H. Wang, "Forecasting integrated circuit output using multivariate grey model and grey relational analysis," Expert Systems with Applications, vol. 36, no. 2, pp. 1403-1409, 2009.

[32] H. Jiang and Y.-C. Hu, J.-Y. Lin and P. Jiang, "Analyzing China's OFDI using a novel multivariate grey prediction model with Fourier series" International Journal of Intelligent Computing and Cybernetics, vol. 12, no. 3, pp. 352-371, 2019.

[33] W. B. Wang and Y. C. Hu, "Multivariate grey prediction models for pattern classification irrespective of time series," Journal of Grey System, vol. 31, no. 2, pp. 135-142, 2019.

[34] Y.-C. Hu, "A multivariate grey prediction model with grey relational analysis for bankruptcy prediction problems," Soft Computing, vol. 24, no. 6, pp. 4259-4268, 2020.

[35] B. Zeng, C. Luo, S. Liu, Y. Bai, and C. Li, "Development of an optimization method for the GM $(1, N)$ model," Engineering Applications of Artificial Intelligence, vol. 55, pp. 353-362, 2016.

[36] H.-T. Pao, H.-C. Fu, and C.-L. Tseng, "Forecasting of CO2 emissions, energy consumption and economic growth in China using an improved grey model," Energy, vol. 40, no. 1, pp. 400-409, 2012.

[37] N. Xu, S. Ding, Y. Gong, and J. Bai, "Forecasting Chinese greenhouse gas emissions from energy consumption using a novel grey rolling model," Energy, vol. 175, pp. 218-227, 2019.

[38] M. Xie, L. Wu, B. Li, and Z. Li, "A novel hybrid multivariate nonlinear grey model for forecasting the traffic-related emissions," Applied Mathematical Modelling, vol. 77, pp. 1242-1254, 2020.

[39] S. Ding, N. Xu, J. Ye, W. Zhou, and X. Zhang, "Estimating Chinese energy-related $\mathrm{CO} 2$ emissions by employing a novel discrete grey prediction model," Journal of Cleaner Production, vol. 259, Article ID 120793, 2020.

[40] Z.-X. Wang and Q. Li, "Modelling the nonlinear relationship between CO2 emissions and economic growth using a PSO algorithm-based grey Verhulst model," Journal of Cleaner Production, vol. 207, pp. 214-224, 2019.

[41] W. B. Wang and Y. C. Hu, "A novel grey residual modification model using neural networks," Journal of Grey System, vol. 30, no. 4, pp. 34-46, 2018.

[42] Y.-C. Hu, "A novel flow-based method using grey relational analysis for pattern classification," International Journal of Information Technology \& Decision Making, vol. 12, no. 1, pp. 75-93, 2013.

[43] S. J. Feng, Y. D. Ma, Z. L. Song, and J. Ying, "Forecasting the energy consumption of China by the grey prediction model," Energy Sources, Part B: Economics, Planning, and Policy, vol. 7, no. 4, pp. 376-389, 2012.
[44] Y.-C. Hu, R.-S. Chen, Y.-T. Hsu, and G.-H. Tzeng, “Grey selforganizing feature maps," Neurocomputing, vol. 48, no. 1-4, pp. 863-877, 2002.

[45] Y. H. Pao, Adaptive Pattern Recognition and Neural Networks, Addison-Wesley, Boston, MA, USA, 1989.

[46] Y.-H. Pao and Y. Takefuji, "Functional-link net computing: theory, system architecture, and functionalities," Computer, vol. 25, no. 5, pp. 76-79, 1992.

[47] G. H. Park and Y. H. Pao, "Unconstrained word-based approach for off-line script recognition using density-based random-vector functional-link net," Neurocomputing, vol. 31, no. 1-4, pp. 45-65, 2000.

[48] Y.-C. Hu, "Grey prediction with residual modification using functional-link net and its application to energy demand forecasting," Kybernetes, vol. 46, no. 2, pp. 349-363, 2017.

[49] D. R. Luo, K. Z. Guo, and H. R. Huang, "Regional economic forecasting combination model based on RAR + SVR," Advances in Intelligent Systems and Computing, Fuzzy Information \& Engineering And Operations Research \& Management, vol. 211, pp. 329-338, 2014.

[50] S. Aminikhanghahi and D. J. Cook, "A survey of methods for time series change point detection," Knowledge and Information Systems, vol. 51, no. 2, pp. 339-367, 2017.

[51] D. Pi, J. Liu, and X. Qin, "A grey prediction approach to forecasting energy demand in China," Energy Sources, Part A: Recovery, Utilization, and Environmental Effects, vol. 32, no. 16, pp. 1517-1528, 2010.

[52] L. Suganthi and A. A. Samuel, "Energy models for demand forecasting-A review," Renewable and Sustainable Energy Reviews, vol. 16, no. 2, pp. 1223-1240, 2012. 